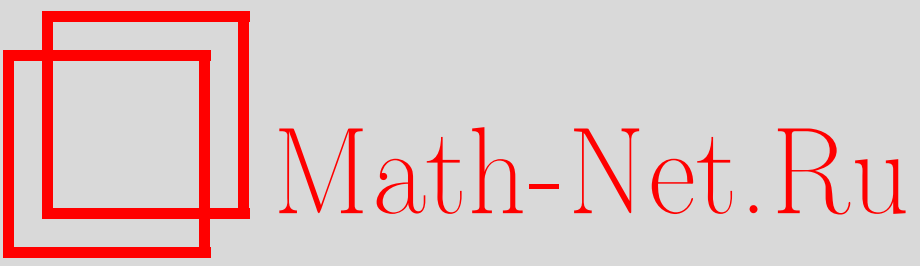

Дж. Гоф, Бозонно-фермионные белые шумы и процесс отражения, ТМФ, 2000, том 124, номер 1, 36-47

DOI: https://doi.org/10.4213/tmf624

Использование Общероссийского математического портала Math-Net.Ru подразумевает, что вы прочитали и согласны с пользовательским соглашением

http://www.mathnet.ru/rus/agreement

Параметры загрузки:

IP : 54.164 .48 .24

26 апреля 2023 г., 06:56:14 
ТЕОРЕТИЧЕСКАЯ

И МАТЕМАТИЧЕСКАЯ

ФИЗИКА

Том 124, № 1

июль, 2000

(C) 2000 г.

Дж. Гоф*

\section{БОЗОННО-ФЕРМИОННЫЕ БЕЛЫЕ ШУМЫ И ПРОЦЕСС ОТРАЖЕНИЯ}

Процесс отражения использован, чтобы объединить бозонное и фермионное квантовые стохастические исчисления. При этом возникают проблемы при использовании стандартного исчисления Стратоновича. Показано, что исчисление Стратоновича с аналитическим продолжением позволяет описать процесс отражения с помощью бозонного белого шума и построить функционал от фермионного белого шума.

\section{1. ВВЕДЕНИЕ}

Теория некоммутативных случайных процессов на бозонном фоковском пространстве, созданная Хадсоном и Партасарати [1], явилась началом нового этапа в исследовании открытых систем, став первой конкретной математической основой для далеко идущего обобщения стандартного стохастического описания классических открытых систем. В рамках этого обобшения можно в явном виде найти унитарные коциклы (т.е. сингулярные возмушения квантовой динамики) для диссипативных эволюций, ассоциированных с произвольным генератором Линдблада [2].

Теория Хадсона и Партасарати создавалась по аналогии со стохастическим исчислением Ито. Хотя такой подход и полезен для математических целей, он затемняет некоторые физические аспекты. Исчисление Ито неудобно для стохастических генераторов квантовых потоков, поскольку в нем не выполняются стандартные формулы дифференцирования произведения и сложной функции, а также скрывается физическая симметрия, присушая исследуемой модели, что затрудняет описание генераторов как стохастических производных.

Обычно это исправляется обращением к исчислению Стратоновича. В работах [3-5] теория квантовых белых шумов была развита комбинированием исчислений Ито и Стратоновича, которые, по сути дела, основываются соответственно на виковом и вейлевом упорядочениях. Пределы слабой связи и низкой плотности [6] служат мотивацией для использования некоммутативной версии теорем Вонга-Закаи или Хасьминского. Отличительной чертой новой теории, присутствуюшей в традиционных подходах лишь неяв-

* Department of Mathematical Physics, National university of Ireland, Maynooth, Ireland. E-mail: jgough@thphys.may.ie 
но, является то, что она позволяет сформулировать аналитически продолженное исчисление Стратоновича.

Такое исчисление основывается, грубо говоря, на некоммутативном обобщении того факта, что приращение процесса $X_{t}$ относительно процесса $Z(t)$ имеет вид

$$
X_{t} d_{S} Z(t) \equiv X_{t}(Z(t+\kappa d t)-Z(t-\bar{\kappa} d t)),
$$

где $\kappa=\gamma / 2+i \sigma$. Если $\sigma=0$, то мы имеем дело со стандартной теорией Стратоновича. Постоянная $\gamma$ положительна и играет роль коэффициента диффузии для шума. Ее можно сделать равной единице.

В этой работе мы обсуждаем физическое значение параметра $\sigma$ и приводим пример процесса отражения [7], для которого преобразование исчисления Ито в исчисление Стратоновича сингулярно при $\sigma=0$.

\section{2. АНАЛИТИЧЕСКОЕ ПРОДОЛЖЕНИЕ ИСЧИСЛЕНИЯ СТРАТОНОВИЧА}

В [3] были введены процессы белого шума $a^{\sharp}(t)$, удовлетворяющие перестановочным соотношениям

$$
\left[a(t), a^{\dagger}(s)\right]=\kappa \delta_{+}(t-s)+\bar{\kappa} \delta_{-}(t-s)=: \Delta_{F}(t-s) .
$$

Правая часть - это распределение $\Delta_{F}(t-s)$ на пространстве кусочно-гладких функций [8]. По определению имеем

$$
\int_{-\infty}^{\infty} \delta_{ \pm}(t-s) f(s) d s=f\left(t^{ \pm}\right)
$$

В частности, $\Delta_{F}$ возникает как предел фейнмановского пропагатора для квантов окружающей среды. Расширение на кусочно-гладкие функции (a, скажем, не на шварцевские) позволяет в качестве пробных функций ввести ступенчатые функции, и, следовательно, (2.1) включает всю информацию, содержашуюся в причинных соотношениях.

Пара $a_{t}$ и $a_{t}^{\dagger}$ является сопряженной на бозонном фоковском пространстве кусочно-гладких пробных функций. Унитарный процесс $U_{t}$ задан как решение задачи Коши для квантового стохастического дифференциального уравнения (КСДУ) $\dot{U}_{t}=-i \Upsilon_{t} U_{t}$, $U_{0}=1$ в вотором стохастический гамильтониан должен быть взят в виде $\Upsilon_{t}=$ $E a^{\dagger}(t) a(t)+F a^{\dagger}(t)+F^{\dagger} a(t)+H$, где $E, F$ и $H$ - ограниченные операторы в фиксированном гильбертовом пространстве $\mathcal{H}_{0}, E$ и $H$ - самосопряженные операторы.

ТЕОрема 1. Процесс $U_{t}$ удовлетворяет перестановочным соотноиениям

$$
\begin{array}{r}
{\left[a(t), U_{s}\right]= \begin{cases}0, & t>s, \\
-i \kappa(E a(t)+F) U_{t}, & t=s, \\
-i \gamma U_{s} U_{t}^{\dagger}(E a(t)+F) U_{t}, & t<s,\end{cases} } \\
{\left[a^{\dagger}(t), U_{s}\right]= \begin{cases}0, & t>s, \\
i \bar{\kappa}\left(E a^{\dagger}(t)+F^{\dagger}\right) U_{t}, & t=s, \\
i \gamma U_{s} U_{t}^{\dagger}\left(E a^{\dagger}(t)+F^{\dagger}\right) U_{t}, & t<s .\end{cases} }
\end{array}
$$


ДокаЗАТЕЛЬСтво. Пусть $T$ обозначает временное упорядочение. Процесс $U_{t}$ может быть записан как

$$
T \exp \int_{0}^{t} d v \Upsilon_{v} U_{v}
$$

и при $t<s$ имеем

$$
U_{s} U_{t}^{\dagger}=T \exp \int_{t}^{s} d v \Upsilon_{v} U_{v}
$$

Можно также записать

$$
U_{t}=1-i \int_{0}^{t} d v \Upsilon_{v} U_{v}
$$

Ясно, что $U_{s}$ не зависит от $a^{\sharp}(t)$ при $s<t$. Заметим, что $\left[a(t), \Upsilon_{v}\right]=(E a(v)+F)[a(t)$, $\left.a^{\dagger}(u)\right]$. При $s=t$ имеем

$$
\left[a(t), U_{t}\right]=-i \int_{0}^{t} d v\left[a(t), \Upsilon_{v}\right] U_{v} .
$$

Отметим, что $\left[a(t), U_{v}\right]=0$ при $t<u$. Из перастановочных соотношений $(2.1)$ следует, что лишь половина распределения дает вклад из-за ограничения области интегрирования. Следовательно, $\left[a(t), U_{t}\right]=-\kappa(E a(t)+F) U_{t}$.

При $t>s$ имеем

$$
\left[a(t), U_{s}\right]=-i \int_{0}^{s} d v\left\{\left[a(t), \Upsilon_{v}\right] U_{v}+\Upsilon_{v}\left[a(t), U_{v}\right]\right\}
$$

В первый член подынтегрального выражения вносят вклад оба $\delta$-распределения из (2.1). Во втором члене мы видим, что область интегрирования в действительности есть интервал $(t, s)$, поскольку коммутатор $\left[a(t), U_{v}\right]$ обращается в нуль при $u<t$. Следовательно,

$$
\begin{aligned}
{\left[a(t), U_{s}\right]=} & -i \gamma(E a(t)+F) U_{t}-i \int_{t}^{s} d v \Upsilon_{v}\left[a(t), U_{v}\right]= \\
= & -i \gamma\left\{1-i \int_{t}^{s} d v_{1} \Upsilon_{v_{1}}+\right. \\
& \left.+(-i)^{2} \int_{t}^{s} d v_{1} \int_{t}^{v_{1}} d v_{2} \Upsilon_{v_{1}} \Upsilon_{v_{2}}+\cdots\right\}(E a(t)+F) U_{t}
\end{aligned}
$$

Если суммировать ряд, то получим $U_{s} U_{t}^{\dagger}$, что и требовалось.

Для второго набора коммутаторов доказательство аналогично.

Свойства $U_{t}$, установленные в теореме 1 , подобны свойствам, которыми обладают входные процессы, исследованные Гардинером в разделе 5.3 работы [9]. Однако эти входные процессы получены из “горячей ванны”, которая имеет спектр, содержащий все вещественные частоты, и соответствуют первому марковскому приближению. Следовательно, подход Гардинера имеет дело только с вешественными значениями $\kappa$. 
ТЕОРемА 2. КСДУ для процесса $U_{t}$ можно представить в виде

$$
\dot{U}_{t}=\left(1, \frac{1}{\sqrt{\gamma}} a^{\dagger}(t)\right)\left(\begin{array}{cc}
-i \widetilde{H}-\frac{1}{2} L^{\dagger} L & L \\
-W L^{\dagger} & W-1
\end{array}\right)\left(\begin{array}{c}
1 \\
\frac{1}{\sqrt{\gamma}} a(t)
\end{array}\right)
$$

$2 \partial e$

$$
W=\frac{1-i \bar{\kappa} E}{1+i \kappa E}, \quad L=-i \sqrt{\gamma} F^{\dagger}(1+i \kappa E)^{-1}, \quad \widetilde{H}=H+F^{\dagger} \frac{\sigma-|\kappa|^{2} E}{1+|\kappa|^{2} E^{2}} F
$$

ДокАЗАТЕЛЬСТво. Заметим, что если выполнить все умножения в уравнении (2.4), то это уравнение станет нормально-упорядоченной формой КСДУ, т.е. со всеми $a^{\dagger}(t)$ слева и $a(t)$ справа. Нормальное упорядочение делается с учетом одновременного коммутационного соотношения

$$
a(t) U_{t}=U_{t} a(t)=(1+i \kappa E)^{-1}\left\{U_{t} a(t)-i \kappa F U_{t}\right\}
$$

Используя это равенство и его эквивалент для $\left[a^{\dagger}(t), U_{t}\right]$, получаем $(2.4)$.

Процесс $\left(X_{t}\right)_{t}$ называется неупреждающим, если $\left[X_{t}, a^{\sharp}(s)\right]=0$ при $s>t$. Нормально-упорядоченная форма КСДУ может быть описана как КСДУ Хадсона-Партасарати при следуюших условиях:

$$
\begin{aligned}
X_{t} \otimes d A_{t}^{0} & \equiv \frac{1}{\gamma} a^{\dagger}(t) X_{t} a(t) d t, & X_{t} \otimes d A_{t}^{\dagger} & \equiv \frac{1}{\sqrt{\gamma}} a^{\dagger}(t) X_{t} d t, \\
X_{t} \otimes d A_{t} & \equiv \frac{1}{\sqrt{\gamma}} X_{t} a(t) d t, & X_{t} \otimes d t & =X_{t} d t,
\end{aligned}
$$

где $A_{t}^{0}$ - консервативный (калибровочный) процесс, $A_{t}^{\dagger}$ и $A_{t}$ - процессы рождения и уничтожения. Таким образом, КСДУ (2.4) приобретают вид

$$
d U_{t}=(W-1) U_{t} \otimes d A_{t}^{0}-W L^{\dagger} U_{t} \otimes d A_{t}^{\dagger}+L U_{t} \otimes d A_{t}-\left(i \widetilde{H}+\frac{1}{2} L^{\dagger} L\right) U_{t} \otimes d t
$$

который, поскольку оператор $W$ из $(2.5)$, очевидно, унитарный и $\widetilde{H}$ самосопряженньй, определяет унитарный квантовый стохастический процесс на фоковском пространстве (с $\mathcal{H}_{0}$ в качестве исходного пространства).

Заметим, что можно обратить $W=W(E)$ и получить равенство

$$
E=-\frac{W-1}{\sigma(W-1)-\frac{i}{2} \gamma(W+1)}
$$

В частности, если $E=0$, то $W=1$. В этой ситуации (восстанавливая $\sigma$ ) имеем уравнение Гейзенберга для $X_{t}:=U_{t}^{\dagger} X U_{t}, \quad X \in B\left(\mathcal{H}_{0}\right)$, вида $\dot{X}_{t}=\dot{U}_{t}^{\dagger} X U_{t}+U_{t}^{\dagger} X \dot{U}_{t}$ или после нормального упорядочения

$$
\dot{X}_{t}=\left(a^{\dagger}(t)+i \bar{\kappa} F_{t}^{\dagger}\right) \frac{1}{i}\left[X_{t}, F_{t}\right]+\frac{1}{i}\left[X_{t}, F_{t}^{\dagger}\right]\left(a(t)-i \kappa F_{t}\right) .
$$


В частности, если принять, что $\left[F, F^{\dagger}\right]=1$, то в силу унитарности имеем $\left[F_{t}, F_{t}^{\dagger}\right]=1$ при всех $t>0$ и, следовательно, $\dot{F}_{t}=-i a(t)-\kappa F_{t}$, откуда

$$
F_{t}=F e^{-\kappa t}+i \int_{0}^{t} d v e^{-\kappa(t-v)} a_{v}
$$

В результате, используя (2.1), получаем

$$
\left[F_{t}, F_{s}^{\dagger}\right]=e^{-\gamma|t-s|} e^{i \sigma(t-s)}
$$

Параметр $\sigma$ представляет собой некоторое частотное слагаемое в автокорреляционной функции для $F_{t}^{\sharp}$. Вообше, $\widetilde{H}=H+\sigma F^{\dagger} F$. Поэтому $\sigma-$ коэффициент сдвига энергии, вызванного радиационным демпфированием системы со стороны шума. С физической точки зрения это причина хорошо известного лэмбовского сдвига.

\section{3. ПРОЦЕСС ОТРАЖЕНИЯ}

Если рассматривать только стандартные представления шума Стратоновича, т.е. с $\sigma=0$, то унитарный оператор $W$ связан с $E$ соотношением

$$
E=-\frac{i}{2} \frac{W-1}{W+1}
$$

Оно, очевидно, сингулярно при $W=-1$. Это означает, что хотя КСДУ Хадсона-Партасарати с $W=-1$ хорошо определено, для него не сушествует стандартного представления Стратоновича. Процесс отражения $J_{t}$ является неупреждаюшим унитарным процессом, заданным как решение КС ДУ

$$
d J_{t}=-2 J_{t} \otimes d A_{t}^{0}, \quad J_{0}=1
$$

Процесс отражения $J_{t}$ был введен в [7] для того, чтобы получить фермионные стохастические переменные из бозонных. Процесс $J_{t}$ называется также процессом четности. Подробное изложение его значения для суперсимметрии имеется в [10]. Процесс отражения может быть явным образом представлен в виде

$$
J_{t}=e^{i \pi A_{t}^{0}}=T e^{-2 A_{t}^{0}}=\Gamma_{+}\left(-\widehat{\chi}_{[0, t]}+\widehat{\chi}_{(t, \infty)}\right) .
$$

Здесь $\Gamma_{+}\left(e^{i \Theta}\right) \equiv e^{i A^{0}(\Theta)}-$ вторичное квантование некоторого унитарного оператора $V=e^{i \Theta} \in L^{2}[0, \infty)$. Процесс $J_{t}$ обладает свойствами $J_{t}^{2}=1,\left[J_{t}, J_{s}\right]=0$ и $J_{t} \Psi=\Psi$, где $\Psi$ - фоковский вакуум.

Стохастические интегралы

$$
F_{t}^{\sharp}:=\int_{0}^{t} J_{v} \otimes d A_{v}^{\sharp}
$$


являются фермионами, т.е. $\left\{F_{s}, F_{t}^{\dagger}\right\}=t \wedge s$. Действительно, Хадсон и Партасарати показали, что $d F_{t}, d F_{t}^{\dagger}$ и $d A_{t}^{0}$ - дифференциалы, которые порождают фермионное исчисление Ито, обобщаюшее (за счет включения консервативного процесса) исчисление из работы [11].

С нашей точки зрения процесс отражения удовлетворяет КС ДУ

$$
\dot{J}_{t}=-2 a^{\dagger}(t) J_{t} a(t) .
$$

Если $a^{\sharp}(t)$ удовлетворяет перестановочным соотношениям $(2.1)$, то, поскольку $W=-1$, мы получаем из $(2.7)$, что $E \equiv-1 / \sigma$. Следовательно, необходимо принять, что $\sigma \neq 0$. Пусть для простоты $\gamma=1$. Уравнение (3.2) в форме Стратоновича имеет вид

$$
\dot{J}_{t}=-\frac{i}{\sigma} a^{\dagger}(t) a(t) J_{t}
$$

ТЕОРема 3. Процесс отражения удовлетворяет перестановочным соотношениям

$$
\begin{array}{r}
{\left[a(t), J_{s}\right]= \begin{cases}0, & t>s, \\
-(1+i 2 \sigma) J_{t} a(t), & t=s, \\
-2 J_{s} a(t), & t<s,\end{cases} } \\
{\left[a^{\dagger}(t), J_{s}\right]= \begin{cases}0, & t>s, \\
(1-2 i \sigma) a^{\dagger}(t) J_{t}, & t=s, \\
-2 J_{s} a^{\dagger}(t), & t<s .\end{cases} }
\end{array}
$$

ДокАЗАТЕЛЬСТво. Процесс $J_{t}$ неупреждающий и поэтому коммутирует с последующими $a^{\sharp}($.$) . При равных временах мы имеем$

$$
\left[a(t), J_{t}\right]=-2 \int_{0}^{t} d v\left[a(t), a^{\dagger}(u) J_{u} a(u)\right]=-2\left(\frac{1}{2}+i \sigma\right) J_{t} a(t) .
$$

При $t<s$ мы рассуждаем, как при доказательстве теоремы 1. Используя КСДУ (3.3), получаем

$$
\begin{aligned}
{\left[a(t), J_{s}\right]=} & -\frac{i}{\sigma}\left[a(t), \int_{0}^{s} d u\left\{\left[a(t), a^{\dagger}(u)\right] a(u) J_{u}+a^{\dagger}(u) a(u)\left[a(t), J_{u}\right]\right\}\right]= \\
= & -\frac{i}{\sigma}\left\{a(t) J_{t}+\int_{t}^{s} d u a^{\dagger}(u) a(u)\left[a(t), J_{u}\right]\right\}= \\
= & -\frac{i}{\sigma}\left\{1+\left(\frac{-i}{\sigma}\right) \int_{t}^{s} d u_{1} a^{\dagger}\left(u_{1}\right) a\left(u_{1}\right)+\right. \\
& \left.+\left(\frac{-i}{\sigma}\right)^{2} \int_{t}^{s} d u_{1} \int_{t}^{u_{1}} d u_{2} a^{\dagger}\left(u_{1}\right) a\left(u_{1}\right) a^{\dagger}\left(u_{2}\right) a\left(u_{2}\right)+\cdots\right\} a(t) J_{t}= \\
= & -\frac{i}{\sigma} T \exp \left(-\frac{i}{\sigma} \int_{t}^{s} d u a^{\dagger}(u) a(u)\right) a(t) J_{t} \equiv \\
\equiv & -\frac{i}{\sigma} J_{s} J_{t} a(t) J_{t} .
\end{aligned}
$$


Умножая получившееся равенство $\left[a(t), J_{s}\right]=-i J_{s} J_{t} a(t) J_{t} / \sigma$ слева на $J_{t}$, получаем $J_{t} a(t) J_{t}=-2 i \sigma a(t)$, и, следовательно, $\left[a(t), J_{s}\right]=-2 J_{s} a(t)$.

Второй набор соотношений выводится аналогично.

СлЕДСТВИЕ. Шумы $a^{\sharp}(t)$ и прочесс отражения $J_{t}$ связаны соотношениями

$$
\begin{array}{ll}
a^{\sharp}(t) J_{s}=+J_{s} a^{\sharp}(t) & (t>s), \\
a^{\sharp}(t) J_{s}=-J_{s} a^{\sharp}(t) & (t<s),
\end{array}
$$

а при равных временах

$$
\begin{aligned}
a(t) J_{t} & =-2 i \sigma J_{t} a(t), \\
J_{t} a^{\dagger}(t) & =2 i \sigma a^{\dagger}(t) J_{t} .
\end{aligned}
$$

\section{4. ФЕРМИОННЫЕ ШУМОВЫЕ ПЕРЕМЕННЫЕ}

В работе [7] показано, что можно получить фермионные переменные из бозонных. Это возможно на уровне квантового белого шума при условии, что мы используем шумовые поля $a^{\sharp}(t)$, аналитически продолженные в точку $\kappa=\gamma / 2+i \sigma$ при $\sigma \neq 0$. Пусть

$$
f(t)=J_{t} a(t), \quad f_{t}^{\dagger}=a^{\dagger}(t) J_{t}
$$

Непосредственные вычисления показывают, что

$$
f(t) f^{\dagger}(s)=J_{t} a(t) a^{\dagger}(s) J_{s}=J_{t} a^{\dagger}(s) a(t) J_{s}+\Delta(t-s) J_{t} J_{s}
$$

Однако $J_{t} a^{\dagger}(s) a(t) J_{s}=-a^{\dagger}(s) J_{t} J_{s} a(t)$, поскольку в силу (3.5) только одна из двух перестановок приводит к изменению знака. Так как $J_{t}$ и $J_{s}$ коммутируют, имеем $f(t) f^{\dagger}(s)=$ $-f^{\dagger}(s) f(t)+\Delta_{F}(t-s) J_{t} J_{s}$, но $\Delta_{F}(t-s) J_{t} J_{s} \equiv \Delta_{F}(t-s) J_{t}^{2}=\Delta_{F}(t-s)$. Следовательно, шумовые переменные $f^{\sharp}(t)$ удовлетворяют антиперестановочным соотношениям

$$
\left\{f(t), f^{\dagger}(s)\right\}=\Delta_{F}(t-s) .
$$

В таком представлении становятся очевидными несколько важных свойств шумов. Вопервых, мы замечаем, что причина, по которой бозонный консервативный процесс служит также фермионным, заключается в том, что для шумов справедливо равенство [7]

$$
f^{\dagger}(t) f(t) d t=a^{\dagger}(t) J_{t} J_{t} a(t) d t=a^{\dagger}(t) a(t) d t
$$

Кроме того, обратные соотношения имеют вид

$$
a(t)=J_{t} f(t), \quad a^{\dagger}(t)=f^{\dagger}(t) J_{t},
$$

т.е. мы можем выразить бозонные шумы через фермионные. 


\section{5. ПЛОТНОСТИ КВАНТОВЫХ ШУМОВ}

Чтобы продемонстрировать, как перестановочные соотношения (2.1) возникают на практике, мы исследуем обшее описание марковских приближений для нескольких типов квантовых открытых систем [12].

Пусть $\mathcal{H}_{S}$ - гильбертово пространство некоторой системы $S$ и $\mathcal{H}_{R}$ - гильбертово пространство резервуара $R$, содержашего систему $S$. Предполагается, что $\mathcal{H}_{R}=\Gamma_{-}\left(\mathcal{H}_{R}^{1}\right)-$ бозонное фоковское пространство над одночастичным пространством $\left(\mathcal{H}_{R}^{1},\langle., .\rangle_{1}\right)$. Пусть для каждого натурального $n$ имеем $\mathcal{K}_{n} \subset H_{R}^{1}$, и $\mathbf{g}=\left(g_{n}\right)_{n}$ - последовательность пробных функций с $g_{n} \in C\left(\mathbf{R}, \mathcal{K}_{n}\right)$. Обозначим символом $A_{R}^{\sharp}($.$) оператор рождения или$ уничтожения в $\mathcal{H}_{R}$. Положим

$$
a_{n}^{\sharp}(\mathbf{g}, t):=A_{R}^{\sharp}\left(g_{N}(t)\right)
$$

$$
A_{n}^{\sharp}(\mathbf{g}, t):=\int_{0}^{t} d s a_{n}^{\sharp}(\mathbf{g}, s)=A_{R}^{\sharp}\left(\int_{0}^{t} d s g_{n}(s)\right) .
$$

Последний интеграл можно понимать в смысле Бохнера.

Выберем семейство $\mathcal{T}$ последовательностей таких, что для каждых $\mathbf{g}, \mathbf{f} \in \mathcal{T}$ сушествует интегрируемая функция $G_{\mathbf{g}, \mathbf{f}}$ такая, что

$$
\left\langle g_{n}(t), f_{n}(s)\right\rangle_{1} \equiv n G_{\mathbf{g}, \mathbf{f}}(n(t-s)) .
$$

В среднем $\langle.\rangle_{0}=\left\langle\Psi_{R}, . \Psi_{R}\right\rangle$ (или в более общем смысле полуторалинейных форм от коллективных когерентных состояний $\Psi_{R}\left(h_{n}\right)$ с $\left.\mathbf{h} \in \mathcal{T}\right)$ операторы $A_{n}^{\sharp}(\mathbf{g}, t)$ сходятся к квантовым винеровским процессам типа Хадсона-Партасарати с ковариацией

$$
\left[A(\mathbf{g}, t), A^{\dagger}(\mathbf{f}, s)\right]=\lim _{n \rightarrow \infty} \int_{0}^{t} d t^{\prime} \int_{0}^{s} d s^{\prime} n G_{\mathbf{g}, \mathbf{f}}\left(n\left(t^{\prime}-s^{\prime}\right)\right)=t \wedge s \gamma_{\mathbf{g}, \mathbf{f}}
$$

где

$$
\gamma_{\mathbf{g}, \mathbf{f}}:=\int_{-\infty}^{\infty} d \tau G_{\mathbf{g}, \mathbf{f}}(\tau)
$$

Однако среднее значение $\left\langle\int_{0}^{t} d A\left(\mathbf{g}, t^{\prime}\right) A^{\dagger}\left(\mathbf{f}, t^{\prime}\right)\right\rangle_{\text {Fock }}$ получается как предел

$$
\begin{aligned}
& \left\langle\int_{0}^{t} d t^{\prime} \int_{0}^{t^{\prime}} d s^{\prime} a\left(g_{n}\left(t^{\prime}\right)\right) a^{\dagger}\left(f_{n}\left(s^{\prime}\right)\right)\right\rangle_{0}= \\
& \quad=\int_{0}^{t} d t^{\prime} \int_{0}^{t^{\prime}} d s^{\prime} n G_{\mathbf{g}, \mathbf{f}}\left(n\left(t^{\prime}-s^{\prime}\right)\right) \rightarrow t \kappa_{\mathbf{g}, \mathbf{f}}^{+}
\end{aligned}
$$

где

$$
\kappa_{\mathbf{g}, \mathbf{f}}^{+}:=\int_{0}^{\infty} d \tau G_{\mathbf{g}, \mathbf{f}}(\tau) .
$$


Аналогичньгм образом находим

$$
\left\langle\int_{0}^{t} A\left(\mathbf{g}, t^{\prime}\right) d A^{\dagger}\left(\mathbf{f}, t^{\prime}\right)\right\rangle_{\text {Fock }} \equiv t \kappa_{\mathbf{g}, \mathbf{f}}^{-},
$$

где

$$
\kappa_{\mathbf{g}, \mathbf{f}}^{-}:=\int_{-\infty}^{0} d \tau G_{\mathbf{g}, \mathbf{f}}(\tau)
$$

Заметим, что $\gamma=\kappa^{+}+\kappa^{-}$.

Вьшесказанное обобшается на произвольные повторные интегралы способом, который можно резюмировать, сказав, что операторы $a_{n}^{\sharp}(\mathbf{g}, t)$ сходятся к процессам белого шума $a^{\sharp}(\mathbf{g}, t)$ с перестановочными соотношениями

$$
\left[a(\mathbf{g}, t), a^{\dagger}(\mathbf{f}, s)\right]=\kappa_{\mathbf{g}, \mathbf{f}}^{+} \delta_{+}(t-s)+\kappa_{\mathbf{g}, \mathbf{f}}^{-} \delta_{-}(t-s),
$$

где $\delta_{ \pm}-$функционалы, введенные в (2.2) на пространстве кусочно-гладких пробных функций. Заметим, что все повторные интегралы могут быть представлены как интегралы от кусочно-гладких пробных функций. Пространство кусочно-гладких функций всюду плотно в гильбертовом пространстве квадратично интегрируемых функций и инвариантно относительно проецирований на компакты. Поэтому они образуют допустимое подпространство в смысле Хадсона-Партасарати и рассматриваемые нами КСДУ зависят от выбора допустимых подпространств. Следовательно, в таком пространстве эволюция стабильна. Соотношения (5.7) содержат всю информацию о хаотических разложениях предельных шумовых процессов.

Вышесказанное можно интерпретировать как квантовую функциональную центральную предельную теорему, сердшевиной которой является сохранение при переходе к пределу формы теоремы Вика для нормального упорядочения в отношении к временному упорядочению.

Пример 1. Пределы слабой связи и низкой плотности. Пусть $\mathcal{H}_{R}^{1}$ сепарабельно и $S_{t}=e^{i \Omega t}$ - унитарный оператор в $\mathcal{H}_{R}^{1}, \Omega$ - самосопряженный оператор в $\mathcal{H}_{R}^{1}$. Пусть $\mathcal{T}$ - пространство всех последовательностей $\mathbf{g}=\left(g_{n}\right)_{n}$ таких, что

$$
g_{n}(t)=\sqrt{n} S_{n t} g,
$$

где $g$ принадлежит фиксированному подпространству $\mathcal{K}$ пространства $\mathcal{H}_{R}^{1}$. Для $\mathbf{g}, \mathbf{f} \in \mathcal{T}$ имеем

$$
\left\langle g_{n}(t), f_{n}(s)\right\rangle_{1}=n\left\langle g, S_{-n(t-s)} f\right\rangle_{1},
$$

в силу чего $G_{\mathbf{g}, \mathbf{f}}(\tau)=\langle g, \exp [-i \Omega \tau] f\rangle_{1}$ и поэтому

$$
\kappa_{\mathbf{g}, \mathbf{f}}^{+}=\int_{0}^{\infty} d \tau\left\langle g, e^{-i \Omega \tau} f\right\rangle_{1}=\left(\kappa_{\mathbf{f}, \mathbf{g}}^{-}\right)^{*} .
$$

Здесь $\gamma_{\mathbf{f}, \mathbf{g}}=\pi\langle g, \delta(\Omega) f\rangle_{1}$ и $\mathcal{K}$ - фиксированное подпространство, так что $\left.\langle., \delta(\Omega)\rangle_{1}\right\rangle_{1}$ при сужении на $\mathcal{K}$ определяет невырожденную полуторалинейную форму. 
Пример 2. Предел сингулярного спаривания. Пусть $\mathcal{H}_{R}^{1}=L^{2}(\mathbf{R})$ и $\mathcal{T}$ - пространство последовательностей $\mathbf{g}=\left(g_{n}\right)_{n}$, где $g_{n}(t) \in L^{2}(\mathbf{R})$ и

$$
g_{n}(t): \omega \mapsto g\left(\frac{\omega}{n}\right) e^{i \omega t},
$$

$g$ имеет интегрируемое преобразование Фурье. Теперь мы имеем ту же схему, что и прежде, но с

$$
G_{\mathbf{g}, \mathbf{f}}(\tau)=\int_{-\infty}^{\infty} d \Omega g(\Omega)^{*} f(\Omega) e^{-i \Omega \tau},
$$

откуда

$$
\kappa_{\mathbf{g}, \mathbf{f}}^{+}=\int_{0}^{\infty} d \tau \int_{-\infty}^{\infty} d \Omega g(\Omega)^{*} f(\Omega) e^{-i \Omega \tau} .
$$

Комплексное демпфирование. Сингулярный предел является специальным случаем предела слабой связи и предела низкой плотности. Чтобы он был нетривиальным, требуется, чтобы спектр энергии был лебегов и состоял из всех вешественных частот $\Omega$. В силу тождества

$$
\int_{0}^{\infty} d \tau e^{-i \Omega \tau}=\pi \delta(\Omega)-i P V \frac{1}{\Omega}
$$

коэффициенты $\kappa_{\mathbf{g}, \mathbf{g}}^{ \pm}$оказываются комплексными, причем

$$
\kappa_{\mathbf{g}, \mathbf{g}}:=\frac{1}{2} \gamma_{\mathbf{g}, \mathbf{g}} \pm i \sigma_{\mathbf{g}, \mathbf{g}} .
$$

Соотношение между слабой связью и пределом сингулярного спаривания обсуждалось в работе [13].

Тот факт, что $\kappa \equiv \gamma / 2+i \sigma$ является, вообше говоря, комплексной величиной, хорошо известен в физической литературе под именем комплексного демпфирования. Как мы видели, для взаимодействий эмиссионно-абсорбционного типа, в которых лишш $F^{\sharp}$ не равно нулю в стохастическом гамильтониане $\Upsilon_{t}$, вешественная часть $\gamma$ дает демпфирование и обусловлена теми квантами резервуара, которые находятся на массовой поверхности, а $\sigma$ выражает радиационный сдвиг энергии, вызванный виртуальными квантами резервуара. Эффект комплексного демпфирования для гармонической функции $F^{\sharp}$ представлен в формуле (2.8). Комплексное демпфирование в равной степени относится и к пределам малой плотности, когда имеет место рассеяние $(E \neq 0)$, но нет эмиссии или абсорбции $(F=0)$.

\section{6. ОБСУЖДЕНИЕ}

Как было впервые отмечено в работе [3], формализмы Ито и Стратоновича в квантовом стохастическом исчислении можно переформулировать как виково и вейлево упорядочения операторов. В работе [4] это было продемонстрировано в специальном случае 
$\kappa=1 / 2$ и в обшем случае - в работах $[14,15]$. Общее рассмотрение базируется на теореме Вика и на замечаниях Хадсона и Стритера [16], относящихся к алгебраическому следствию викова упорядочения в квантовом стохастическом анализе.

Первая трактовка квантовых стохастических явлений, используюшая исчисление Стратоновича, была предпринята фон Вальденфельсом [17]. Он исследовал эмиссионно-абсорбционное КС ДУ. Затем Гардинер [9] исследовал входные процессы, которые управляют эмиссионно-абсорбционными КСДУ, и разработал подходы Ито и Стратоновича. Он рассматривал, главным образом, уравнения в пределе сингулярного спаривания и вешественной константы $\kappa$. Это эквивалентно так называемому первому марковскому приближению.

Явный вид предельного КСДУ для унитарных эволюций, получающихся для эмиссии-абсорбции (предел слабого спаривания) и для рассеяния (предел низкой плотности), известен довольно давно [12]. Такие уравнения включают последовательности пробных функций, которые приводят к комплексным коэффициентам $\kappa$ и которые, как упомянуто в [3], удобно описывать в терминах белых шумов, удовлетворяюших причинным перестановочным соотношениям типа (2.1).

Чеботарев [18] представил теорию стандартных $(\kappa=1 / 2)$ процессов Стратоновича, к которой сводится уравнение (2.4), выступаюшее как соотношение между операторными коэффициентами, полученными в слабом и сильном резольвентном пределах. Здесь проявляется предел сингулярного спаривания, что должно привести к комплексной константе $\kappa$. Анализ Чеботарева, однако, не вполне корректен, и его главная теорема не включает комплексное демпфирование. Результаты Чеботарева могут быть верными, только если последовательности пробных функций удовлетворяют дополнительным условиям, в силу которых константа $\kappa$ оказывается вешественной. Итак, Чеботарев не смог предложить правильное КСДУ Ито для стохастического предела в специальных случаях слабого спаривания или низкой плотности [12]. Главный дефект - это использование леммы 3.1 из [12], в которой делается незаконный предельный переход под знаком интеграла, что приводит только к вешественному коэффициенту демпфирования, как в (5.3), вместо надлежашего комплексного коэффициента, как в (5.5).

\section{Список литературы}

[1] R. L. Hudson, K. R. Parthasarathy. Commun. Math. Phys. 1984. V. 93. P. 301-323.

[2] G. Lindblad. Commun. Math. Phys. 1976. V. 48. P. 19-30.

[3] Дже. Гох. ТМФ. 1997. Т. 111. № 2. С. 218-233.

[4] Джс. Гох. ТМФ. 1997. Т. 113. № 2. С. 276-284.

[5] J. Gough. Asymptotic Stochastic Transformations for Non-Linear Quantum Dynamical Systems (to appear Rep. Math. Phys.).

[6] L. Accardi, J. Gough, Y. G. Lu. Rep. Math. Phys. 1995. V. 36. № 2/3. P. 155-187.

[7] R.L. Hudson, K. R. Parthasarathy. Commun. Math. Phys. 1986. V. 104. P. 457-470.

[8] J. Dieudonné. Foundations of Modern Analysis. New York: Academic Press International Edition, 1968.

[9] C. W. Gardiner. Quantum Noise. New York: Springer-Verlag, 1991. 
[10] T. M. W. Eyre. Quantum Stochastic Calculus and Representations of Lie Super Algebras. Lecture Notes in Mathematics 1692. Berlin: Springer, 1998.

[11] D. Applebaum. Commun. Math. Phys. 1984. V. 96. P. 473-496.

[12] L. Accardi, Y. G. Lu, A. Frigerio, R. Alicki. An invitation to the weak coupling and low density limit. In: Quantum Probability and Related Topics VI. Ed. L. Accardi. Singapore: World Scientific, 1991. P. 3-31.

[13] L. Accardi, A. Frigerio, Y. G. Lu. Acta Appl. Math. 1992. V. 26. P. 197-208.

[14] J. Gough. Asymptotic Stochastic Transformations for Non-linear Quantum Dynamical Systems. (to appear in Rep. Math. Phys.).

[15] J. Gough. Potential Anal. 1999. V. 11. № 3. P. 213-233.

[16] R. L. Hudson, R. F. Streater. Phys. Lett. A. 1981. V. 86. P. 277.

[17] W. von Waldenfels. Ito Solution of the Linear Quantum Stochastic Differential Equation describing Light Emission and Absorption. In: Lect. Notes in Math. V. 1055. Quantum Probability and Aplications to the Quantum Theory of Irreversible Processes. Proc. Int. Workshop Held at Villa Mondragone, Italy, Sept. 6-11, 1982. Eds. L. Accardi, A. Frigerio, V. Gorini. Berlin: Springer, 1984. P. 384-411.

[18] А. М. Чеботарев. Матем. заметки. 1997. Т. 61. № 4. С. 612-622.

Поступила в редакцию 10.XII.1998 г., после доработки 8.IV.1999 г. 